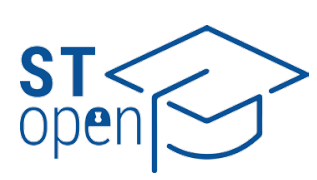

(c) 2021 The Author(s)

ST-OPEN @ 2021

\title{
Regulation of the pleiotropic drug resistance transcription factors Pdr1 and $\mathrm{Pdr} 3$ in yeast
}

\section{Tea Vasiljević ${ }^{1}$ (1), Markus Proft ${ }^{2}$ (1)}

'Bonn-Rhein-Sieg University of Applied Sciences, Department of Natural Sciences, Rheinbach, Germany

${ }^{2}$ Instituto de Biomedicina de Valencia IBV-CSIC, Department of Molecular and Cellular Pathology and Therapy, Valencia, Spain
Correspondence to:

Tea Vasiljević

Ruđera Boškovića 23, 21000 Split

teavasiljevic1@gmail.com

\section{Cite as:}

Vasiljević T, Proft M. Regulation of the pleiotropic drug resistance transcription factors Pdr1 and Pdr3 in yeast. ST-OPEN. 2021; 2: e2021.1911.29.

DOI

https://doi.org/10.48188/so.2.7
Aim: To understand how transcriptional factors Pdr1 and Pdr3, belonging to the pleiotropic drug resistance system, are activated, and regulated after introducing chemical toxins to the cell in the model organism Saccharomyces cerevisiae.

Methods: Series of molecular methods were applied using different strains of $S$. cerevisiae over-expressing proteins of interest as a eukaryotic cell model. The chemical stress introduced to the cell is represented by menadione. Results were obtained performing protein detection and analysis. Additionally, the regulation of the DNA binding of the transcriptional activators after stimulation is quantified using chromatin immunoprecipitation, employing epitope-tagged factors and real-time qPCR.

Results: Our results indicated higher expression levels of the Pdr1 transcriptional factor, compared to its homologous Pdr3 after treatment with menadione. The yeast-cell defence system was tested against various organic solvents to exclude the possibility of their presence potentially affecting the results. The results indicate that Pdr1 is most abundant after 30 minutes from the beginning of the treatment, compared with 240 minutes after the treatment when the function of the transcription factor is faded. It appears that Pdr1 binding to the PDR5 and SNQ2 promoters, which are both activated by Pdr1, peaks around the same time, or more precisely after 40 minutes from the start of the treatment.

Conclusion: The tendency of Pdr1 reduction after its activation by menadione is detected. One possibility is that Pdr1, after recognizing the xenobiotic menadione, is removed by a degradation mechanism. Given the fact that Pdr1 directly binds the xenobiotic molecule, its destruction might help the cells to remove toxic levels of menadione. It is possible that overexpressing the part of Pdr1 which recognizes menadione alone was sufficient to detoxify and hence produce a tolerance towards menadione. 


\section{Introduction}

Eukaryotic cells have developed mechanisms to efficiently respond to xenobiotics. It enables them to tolerate and resist the toxicity of a wide variety of compounds, not related either functionally or structurally, such as toxins or drugs. This ability is known as pleiotropic drug resistance (PDR) or multidrug resistance (MDR). It is found in many organisms from bacteria to mammals. The main mechanism causing this multidrug resistance is the overexpression of membrane transporters (Sá-Correia, dos Santos, Teixeira, Cabrito, \& Mira, 2009). This condition allows the cell to survive toxic exposure. It appears as one of the main problems in chemotherapy treatments in cancer, fungal infections, or neurological pathologies such as epilepsy or depression. It is a product of the transporter's decrease in efficiency (Prasad \& Rawal, 2014; Löscher \& Potschka, 2005). In 1973, point mutations were first described in Saccharomyces cerevisiae affecting the pleiotropic drug resistance. The result of the mutation was greater resistance to several inhibitors with different structures and different modes of action (Rank \& Bech-Hansen, 1973). Consequently, cells have more than one transcriptional factor (TF) that recognizes foreign chemical compounds. Various transporters play a role in this two-step procedure of recovering the cell. The first is recognition and creation of defence, and the second is the processing and getting rid of the toxin by transporting it out of the cell (Figure 1). Eukaryotic cells have many TFs and transporters working on this process of molecule recognition. Membrane transporters of interest belong to the ATP-binding cassette (ABC) group. The expression of many ABC transporters is firmly controlled by TFs within the PDR network (Jungwirth \& Kuchler, 2006).

Transcription factors, proteins that bind to DNA and regulate gene expression by promoting or suppressing transcription, are one of the most abundant classes of proteins in the yeast genome. Most proteins in S. cerevisiae included in transcriptional regulation belong to the zinc cluster subfamily or binuclear proteins. They have zinc fingers of the $\mathrm{Zn}_{2} \mathrm{Cys}_{6}$ type associated with DNA recognition. The well-characterized Gal4p protein is one of the most prominent members of this family (Akache, Wu, \& Turcotte, 2001). Regarding the transcriptional role, Pdr1 is a principal regulator of PDR genes (Balzi, Chen, Ulaszewski, Capieaux, \& Goffeau, 1987). Pdr1 binds to the pleiotropic drug response element (PDRE) motifs at promoter DNA. The action is stimulated by recognition of a toxin and some basal actions. This leads to the conclusion that Pdr1 binding to target promoters is merely not dependent on the chemical stress stimulus.

Pdr1 TF is a main regulator playing an essential role in drug response. Besides Pdr1, another transcriptional factor established in yeast - Pdr3, was also discovered to contribute to multidrug resistance. Pdr3 triggers the export of toxic substances via the activated expression of the $\mathrm{ABC}$ transporter. This mechanism of removing damage from the cell allows the cell to survive xenobiotic stress (Figure 1) (Balzi et al., 1987; Mamnun, Pandjaitan, Mahé, Delahodde, \& Kuchler, 2002). It was concluded that both Pdr1 and Pdr3, control the transcription of various genes encoding drug exporters in the plasma membrane (Balzi \& Goffeau, 1995; Katzmann, Burnett, Golin, Mahe, \& Moye-Rowley, 1994). The first target of Pdr1 was found by the overexpression of a DNA fragment able to confer MDR in Saccharomyces cerevisiae (Leppert et al., 1990). The PDR gene included in this DNA frag- 


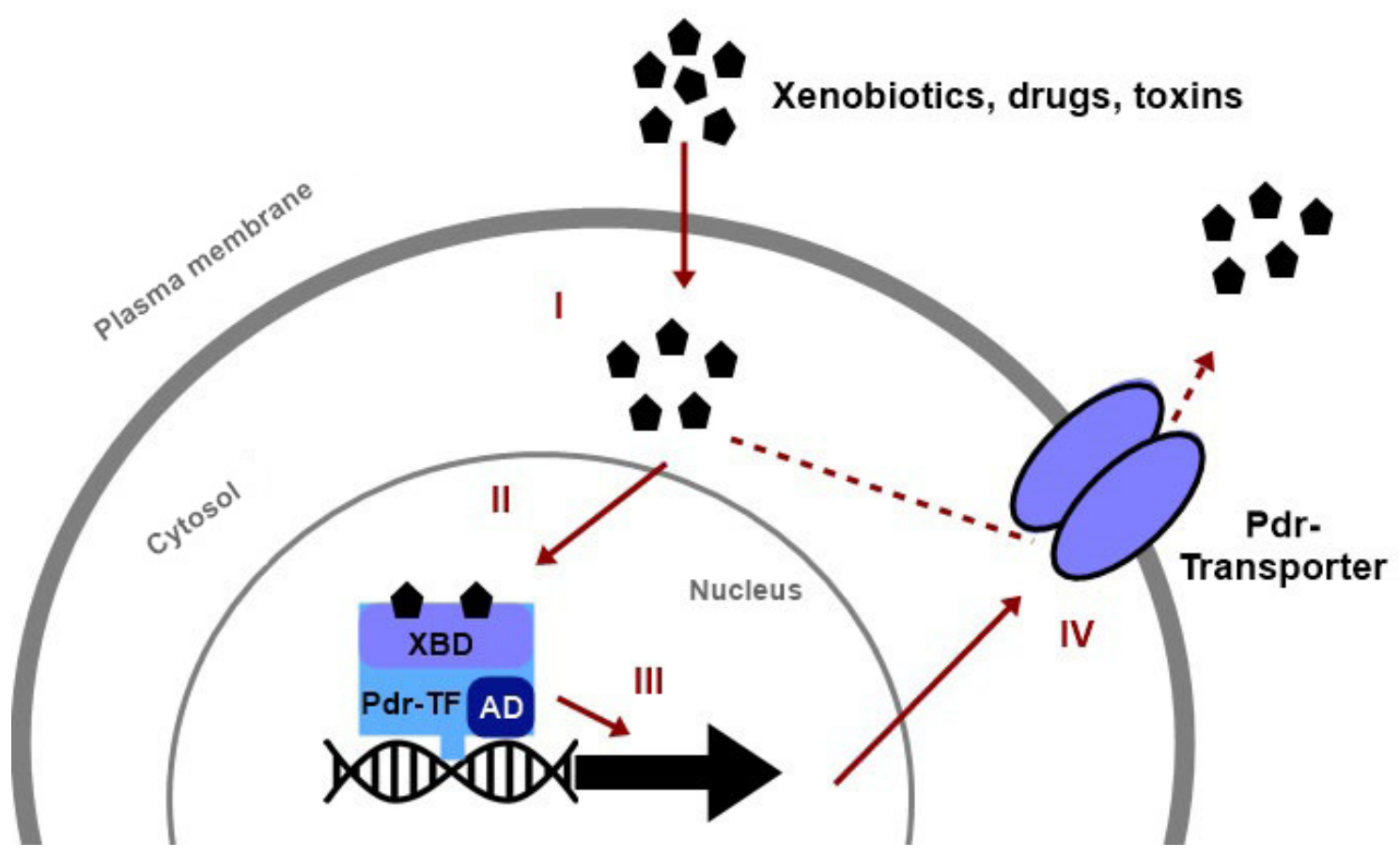

Figure 1. Scheme of the pleiotropic drug resistance (PDR) system. (I) Different compounds with xenobiotic activity are entering the cell. (II) The chemical compounds are directly recognized by specialized transcription factors. (Pdr-TF are specific transcriptional factors of the PDR system). Those TFs contain certain domains for xenobiotic recognition. (XBD = Xenobiotic Binding Domain), or for the activation of transcription (AD = Activation domain). (III) The binding product of the xenobiotic activates Pdr-TF which results in increased expression of the genes in charge of the PDR transporters system. (IV) Activation of those transporters in the plasma membrane exports xenobiotics outside of the cell.

ment is PDR5, the deletion of which conferred drug hypersensitivity (Meyers et al., 2006). This points out that the PDR1 gene encodes a transcription factor that targets the PDR5 gene, as a new member of the $\mathrm{ABC}$ family.

PDR transporters expression is managed by multiple TFs. As mentioned before, TFs with particular interest for this work are Pdr1 (Balzi et al., 1987) and Pdr3 (Delaveau, Delahodde, Carvajal, Subik, \& Jacq, 1994). They are considered functional homologues. Pdr1 and Pdr3 regulate many genes, encoding for example PDR5, PDR15, SNQ2 and YOR1. Pdr3 particularly encodes the PDR3 gene (Balzi \& Goffeau, 1995). Additionally, Pdr1 and Pdr3 can positively or negatively affect the expression of target genes, suggesting that also other factors regulate their activity (Jungwirth \& Kuchler, 2006). Among those two TFs, Pdr1 has a stronger phenotype for drug response when compared to Pdr3. However, the Pdr3 is yet pivotal for PDR responses (Katzmann et al., 1994). Therefore, based on the data already available and by applying adapted molecular methods, the aim was to gain a detailed insight into the defence system and function of PDR TFs Pdr1 and Pdr3 in yeast. It was achieved by inducing the transcription using menadione and other chemical toxins.

Menadione (2-Methyl-1,4-Naphthalenedione), also known as the "Vitamin K3" is a fat-soluble vitamin, synthetic naphthoquinone that does not contain the isoprenoid side chain and biological action, but can be converted to active vitamin $\mathrm{K} 2$, menaquinone, after alkylation in vivo (in the liver). Menadione has been used largely as an oxidant stressor in the cells. It is a very effective molecule to activate multidrug response in yeast cells. The oxidative stress that menadione causes in yeast is regulated by the level of transcription. Menadione is connected to protein oxidation and disruption of homeostasis. 


\section{Materials and methods}

\section{Yeast strains and cultures}

S. cerevisiae strains used in the experiments are listed in Table 1. The growth of yeast strains was performed using Yeast Extract Peptone Dextrose (YPD) medium and the Synthetic Dextrose (SD) medium. The YPD medium was composed of $1 \%$ yeast extract (CONDA, Madrid, Spain), 2\% bacteriological peptone (Condalab, Madrid, Spain) and 2\% glucose (dextrose) (ITW Reagents, Chicago, USA), dissolved in MiliQ water. The SD culture medium was composed of $0.7 \%$ yeast nitrogen base (YNB) (Condalab, Madrid, Spain), $2 \%$ glucose, and $50 \mathrm{mM}$ succinic acid pH = 5.5 (Merck, Darmstadt, Germany), dissolved in MiliQ water. In addition, amino acids and nitrogenous bases ( $25 \mu \mathrm{g} \mathrm{ml}^{-1}$ adenine, $100 \mu \mathrm{g}$ $\mathrm{ml}^{-1}$ leucine and $25 \mu \mathrm{g} \mathrm{ml}^{-1}$ uracil) were added to this medium. In solid media, in both cases, $2 \%$ bacteriological agar was added.

Table 1. List of $S$. cerevisiae strains used for the experiments with genotype and the origin

\begin{tabular}{|c|c|c|}
\hline \multicolumn{3}{|c|}{ S. cerevisiae } \\
\hline Strain & Relevant genotype & Source \\
\hline BY4741 & $\begin{array}{l}\text { WT, MATa; his3 } \Delta 1 \text {; leu2 } \Delta 0 \text {; met } 15 \Delta 0 \text {; } \\
\text { ura3 } \Delta 0\end{array}$ & \#162 laboratory strain collection \\
\hline BY4741 Pdr1-myc & BY4741 with PDR1-2xmyc::KAN & \#1905 laboratory strain collection \\
\hline BY4741 Pdr1-HA & BY4741 with PDR1-3xHA::KAN & \#1907 laboratory strain collection \\
\hline BY4741 Pdr3-myc & BY4741 with PDR3-2xmyc::KAN & $\# 1909$ laboratory strain collection \\
\hline BY4741 Pdr3-HA & S.c. BY4741 with PDR3-3xHA::KAN & \#1911 laboratory strain collection \\
\hline W303-1A-Gal4-Pdr1 & $\begin{array}{l}\text { W303-1A with plasmid pGBKT7-Gal4(DB- } \\
\text { D)-PDR1 }\end{array}$ & \#1855 laboratory strain collection \\
\hline W303-1A-Gal4-Pdr3 & $\begin{array}{l}\text { W303-1A with plasmid pGBKT7-Gal4(DB- } \\
\text { D)-PDR3 }\end{array}$ & \#1857 laboratory strain collection \\
\hline W303-1A-Gal4-Stb5 & $\begin{array}{l}\text { W303-1A with plasmid pGBKT7-Gal4(DB- } \\
\text { D)-STB5 }\end{array}$ & \#1865 laboratory strain collection \\
\hline
\end{tabular}

\section{Preparation of menadione stocks}

A $50 \mathrm{mM}$ menadione stock, a strong oxidant, which was used to cause chemical stress and PDR responses in yeast (Chen, Miyazaki, Tsai, \& Bennett, 2007; Schnell, Krems, \& Entian, 1992), was prepared by diluting $86.09 \mathrm{mg}$ of solid menadione in $10 \mathrm{ml}$ of ethanol or in 10 $\mathrm{ml}$ of dimethyl sulfoxide (DMSO) (ITW Reagents, Chicago, USA).

\section{Preparing cells for immunodetection}

The expression levels and integrity of the Pdr1 and Pdr3 proteins were determined by anti-HA and anti-myc immunodetection. For precultures $3 \mathrm{ml}$ of YPD is supplemented with $12 \mu \mathrm{l}$ of G418 from $250 \mathrm{x}$ stock. Measuring OD600 with target value around 1 A, diluted 1:5 with distilled water and YPD used as blank. Total protein from whole cell extracts were obtained by glass bead lysis in Extraction buffer (Buffer A) (50 mM Tris/HCl pH=7.5, $150 \mathrm{mM}$ $\mathrm{NaCl}, 15 \mathrm{mM}$ EDTA, 0.1 \% Triton X-100, 2 mM DTT, 1mM PMSF) supplemented with protease inhibitors (Pierce ${ }^{\mathrm{TM}}$, Protease Inhibitor Mini, EDTA-free (Thermo Scientific, Waltham, MA, USA)) using the Precellys ${ }^{\circledR}$ Evolution homogenizer (Bertin Technologies, France). The 
extracts were resuspended in 2x Laemmli buffer (120 mM Tris/HCl pH 6.8; 3\% SDS; $40 \mathrm{mM}$ DTT; $4 \mathrm{mM}$ EDTA; $12 \%$ sucrose; $0.1 \mathrm{mg} / \mathrm{ml}$ bromophenol blue) and heated for $5 \mathrm{~min}$ at $95^{\circ} \mathrm{C}$. Using the BIORAD Mini Protean equipment (Bio-Rad, USA), proteins were separated by $8 \%$ SDS-PAGE Resolving gel, with preceded 3\% Stacking gel: and analyzed by immunoblotting on PVDF membranes.

\section{Preparation and detection of antibodies}

For detection of the HA tagged epitopes of the TFs, a-HA mouse monoclonal antibody (12CA5, ROCHE 1:5000) and a-mouse monoclonal secondary antibody (NA931, SIGMA 1:10000) were used. The bands were visualized and detected by fluorescence using the ECL kit; ECLTM Prime Western Blotting Detection System (GE Healthcare - Amersham Biosciences) and quantified with ImageQuant LAS-4000 mini system and software programme. DB71 staining of the membranes was applied as a loading control and visualisation (Hong, Yoo, \& Choi, 2000). For the myc epitope tagged TFs, primary antibody used was anti-myc mouse monoclonal antibody (9E10, ROCHE 1:5000) and secondary a-mouse monoclonal antibody (1:10000) ditto.

\section{Protein immunoprecipitation}

$2 \mathrm{mg}$ of total protein was mixed with $2 \mu \mathrm{l}$ monoclonal anti-HA or anti-myc antibody. Metal beads (Dynabeads ${ }^{\mathrm{TM}}$ Protein $\mathrm{G}$ for Immunoprecipitation, Invitrogen by Thermo Fisher Scientific) were added to the samples. As the epitope binds to the constant part of the antibody, the proteins of interest stay bound to magnetic beads and the undesired unspecific proteins are eliminated by selection with a DynaMag (Thermo Fisher Scientific) for separation.

\section{Protein quantification}

Bio-Rad Protein Assay used for concentration determination of the solubilized protein. The assay includes addition of an acidic dye to the protein solution, and subsequent measurement of the absorbance at $595 \mathrm{~nm}$ with a spectrophotometer or microplate reader. Comparison to a standard curve prepared with BSA provides a relative measurement of protein concentration. Colour change occurs in response to different protein concentrations. In the present work, the Microtiter Plate protocol was applied. Dye reagent (Bio-Rad Protein Assay Dye Reagent Concentrate) is prepared by diluting one part of Dye Reagent Concentrate (Coomassie blue) with 4 parts of water. Samples were prepared in duplicates.

\section{Chromatin immunoprecipitation assay}

Chromatin immunoprecipitation was performed according to the previously described protocols (Kuras \& Struhl, 1999).

Preparation of crosslinked chromatin (Kuras \& Struhl, 1999) for $40 \mathrm{ml}$ yeast culture. Addition of $37 \%$ formaldehyde to the exponentially grown culture for the final formaldehyde concentration of $1 \%$. After the incubation at room temperature, $3 \mathrm{M}$ glycine is added. Cells are washed with cold TBS (20 mM Tris/HCl pH 7.5, $150 \mathrm{mM} \mathrm{NaCl}$ ) and cold FA 
lysis buffer (50 mM Hepes/KOH pH 7.5, 150 mM NaCl, 1 mM EDTA, 1\% Trion X-100, 0.1\% sodium deoxycholate, $1 \mathrm{mM}$ PMSF). $0.1 \%$ SDS is added freshly. Cells were again disrupted with glass beads with the Precellys lysing machine (Bertin Instruments, France). With the collection of pellets chromatin was obtained. Sonication of the samples was done with UP200S sonicator (Hielscher Ultrasonics GmbH, Germany), to get an average of $300 \mathrm{bp}$ DNA fragments by 6 cycles of 15 seconds with ice in between. Soluble chromatin fragments accumulate in the supernatant.

Immunoprecipitation (IP), decrosslink, and analysis by real-time PCR. $400 \mu \mathrm{l}$ of total chromatin sample is incubated with antibodies. Again, the metal Protein $G$ beads were added into a sample, incubated $90 \mathrm{~min}$ at room temperature on a roller. When finished, washing steps are performed in the following order: first, beads are resuspended in $250 \mu \mathrm{l}$ buffer C (50 mM Tris/HCl pH 7.5, $10 \mathrm{mM}$ EDTA, 1 \% SDS) and eluted by incubation for $10 \mathrm{~min}$ at $65^{\circ} \mathrm{C}$ using a heated shaker at maximum speed. After centrifugation, samples with "IP chromatin” were mixed with $250 \mu \mathrm{l} 1 \mathrm{x}$ TE (10 mM Tris/HCl pH 8.0, $1 \mathrm{mM}$ EDTA $)+20 \mu \mathrm{l}$ Pronase (Roche, Switzerland) (20 mg ml-1). In this step, samples with "total chromatin" (without IP) were prepared as well. Decrosslink of the samples is done for 1 hour at $42^{\circ} \mathrm{C}$ followed by 5 hours at $65^{\circ} \mathrm{C} .50 \mu \mathrm{l}$ of $4 \mathrm{M} \mathrm{LiCl}$ was added and samples were extracted once with phenol/chloroform/isoamylalcohol and once with chloroform/isoamylalcohol. DNA is precipitated with $1 \mathrm{ml}$ ethanol $+20 \mu$ g glycogen (Roche, Switzerland). Samples were incubated overnight at $-20^{\circ} \mathrm{C}$ and the next day washed with $96 \%$ ethanol and resuspended in $100 \mu \mathrm{l} \mathrm{TE}$. Preparation of beads: a day before use, $20 \mu \mathrm{l}$ of beads per IP sample are taken and washed with centrifugation (20 seconds, $1000 \mathrm{rmp}$ ) and supernatant discarded using the magnet mentioned before. Subsequent washes are done with $1 \mathrm{ml}$ PBS ( $1 \mathrm{x}$ PBS $=140$ $\mathrm{mM} \mathrm{NaCl}, 2.7 \mathrm{mM} \mathrm{KCl}, 10 \mathrm{mM} \mathrm{Na}_{2} \mathrm{HPO}_{4}, 1.8 \mathrm{mM} \mathrm{KH}_{2} \mathrm{PO}_{4}$, adjusted to $\mathrm{pH} 7.3$ and autoclaved; supplemented with BSA immediately before use) and $5 \mathrm{mg} \mathrm{ml}^{-1} \mathrm{BSA}$ mix. $2 \mu \mathrm{l}$ of anti-HA was added per IP sample and incubated at a roller overnight at $4^{\circ} \mathrm{C}$. The next day; centrifugation and wash steps were repeated. At last, beads are resuspended in the original volume using PBS $+5 \mathrm{mg} \mathrm{ml}^{-1} \mathrm{BSA}$ mix and used for IPs. Reactions are run in triplicates in 96 well plates in real time PCR machine (Applied Biosystems 7500, Termo Fisher, Waltham, MA, USA). Plates were covered with adhesive covers and program is being run: 1 cycle 2 min at $95^{\circ} \mathrm{C}$ and 40 cycles of: 5 seconds $95^{\circ} \mathrm{C}, 5$ seconds $53^{\circ} \mathrm{C}$ and 30 seconds $72{ }^{\circ} \mathrm{C}$. System used is 7500 FAST Real-Time PCR system and the software 7500 v2.3 (Applied Biosystems, Thermo Fisher Scientific, Waltham, MA, USA).

For the quantitation of DNA fragments, quantitative PCR (qPCR) was performed with the sample preparations using either $3 \mu \mathrm{l}$ of IP undiluted sample per reaction or $3 \mu \mathrm{l}$ of 1:10 diluted total chromatin sample. The PCR reaction (20 $\mu$ l final volume) contains: $3 \mu \mathrm{l}$ template DNA (IP or total protein), $6 \mu \mathrm{l}$ primer $1+2$ (from $3.3 \mu \mathrm{M}$ stock) (Table 2), $10 \mu \mathrm{l} \mathrm{EVA}$ Green master mix (adjusted for number of samples: $800 \mu \mathrm{l} 2 \times \operatorname{mix}+9.6 \mu \mathrm{l} \mathrm{ROX}(0.1 \mathrm{mM})$ ).

Table 2. Primers used for real time qPCR following the combination of: PRIMER A + PRIMER B as described in text above

\begin{tabular}{llcl} 
& \multicolumn{3}{c}{ Primer name + laboratory serial lab } \\
PRIMER A & POL1 +1796; \#159 & SNQ2 -685; \#1261 & PDR5 -380; \#1257 \\
PRIMER B & POL1 +1996; \#160 & SNQ2 -495; \#1262 & PDR5 -243; \#1258 \\
\hline
\end{tabular}




\section{Growth assay}

The W303-1A strains mentioned in Table 1 were used in this experiment. W303-1A = (his), leu, (trp), ura, ade; are amino acids needed for the growth. Strains were seeded on SD + leu + ura + ade solid agar plates and incubated at $30^{\circ} \mathrm{C}$ overnight. After addition of MEN to the samples, the culture aliquots are introduced into an automated microplate reader and growth is constantly monitored for 72 hours. The system used for growth and Bradford assay as well is a Tecan Spark microplate reader platform with the Spark Control Magellan 2.2. software.

\section{Results}

\section{Detection of Pdr1 and Pdr3: Effect of menadione treatment}

Samples of precultures prepared from the wild type, Pdr1, and Pdr3 over-expressing cells were performed in this experiment. Exponentially grown cultures were treated with ethanol alone or menadione dissolved in ethanol (Figure 2).

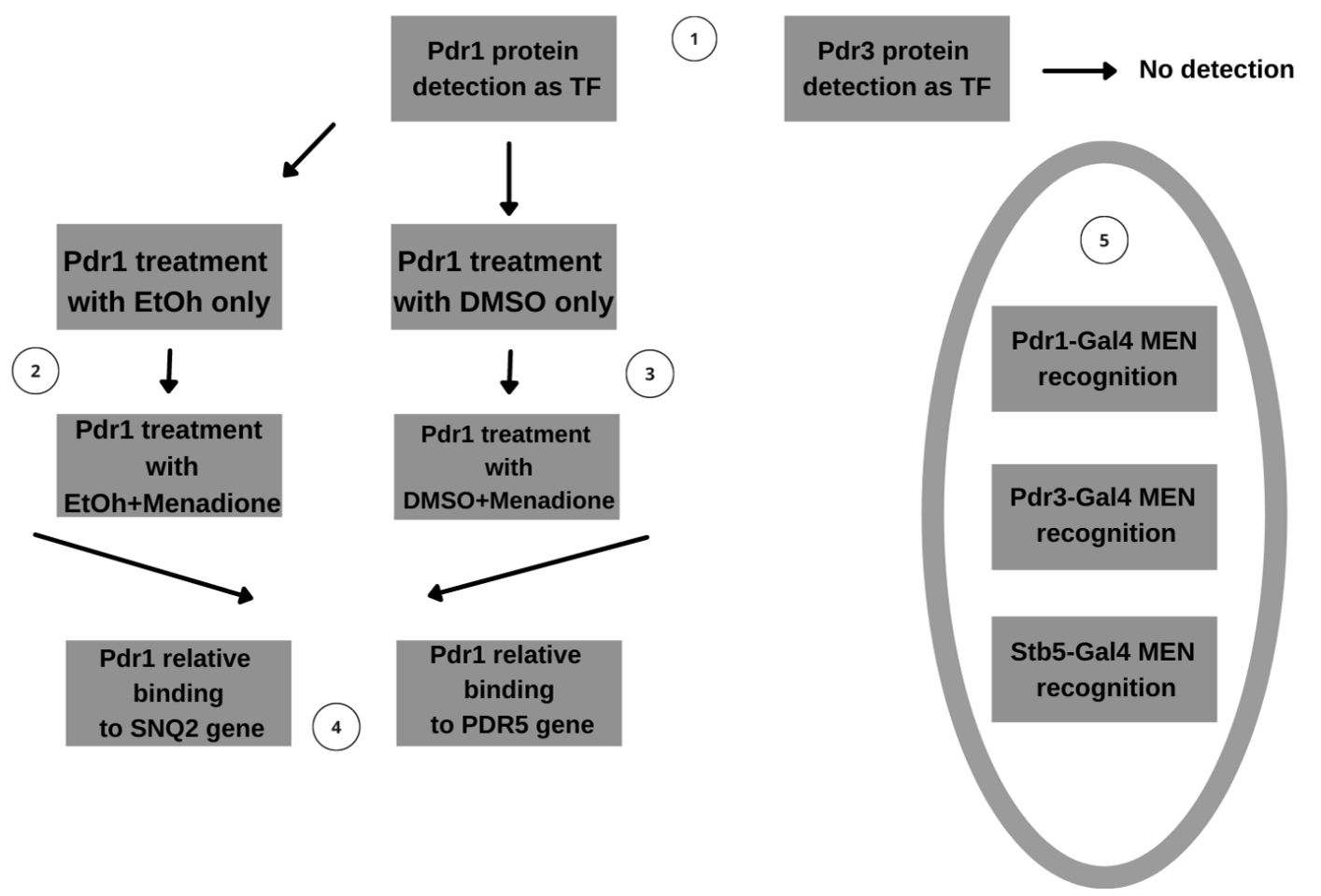

Figure 2. The experimental part of the thesis began with testing Pdr1 and Pdr3 TFs protein detection. The results indicated higher expression levels of the Pdr1 transcriptional factor, compared to its homologous Pdr3 after treatment with menadione (1). Pdr1 granted the required reaction therefore was taken for further experiments regarding menadione. To exclude the possibility of their presence potentially affecting the results, the yeast-cell defence system was tested against various organic solvents (ethanol and DMSO). $(2,3)$ The results indicated that the Pdr1 is mostly abundant at certain time points. Regarding that information, the next step was to test the level of Pdr1 bound to DNA using chromatin immunoprecipitation. It was proven that the selected genes PDR5 and SNQ2 are both targeted by Pdr1 in the promoter region. (4) In addition, to test the hypothesis, hybrids with replaced DNA binding domains were used to detect level of recognized menadione (MEN). 
The results which represent the total protein sample were shown in Figure 3. The results of immunoprecipitation outcomes of samples loaded and separated in SDS gels and transferred to membranes using electrophoresis are visible in Figure 4.

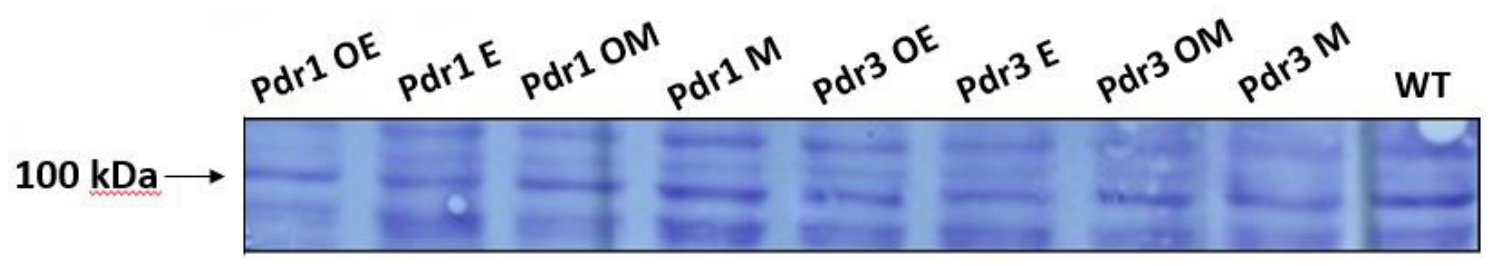

Figure 3. Results of electrophoresis, total cell protein load visualized on PVDF membrane with DB71 staining. Proteins bands were visible in the molecular weight range indicated at around $100 \mathrm{kDa}$. $\mathrm{OE}=\mathrm{ethanol}$ control, E=ethanol treatment, $\mathrm{OM}=$ menadione control, $\mathrm{M}=$ menadione + ethanol treatment.

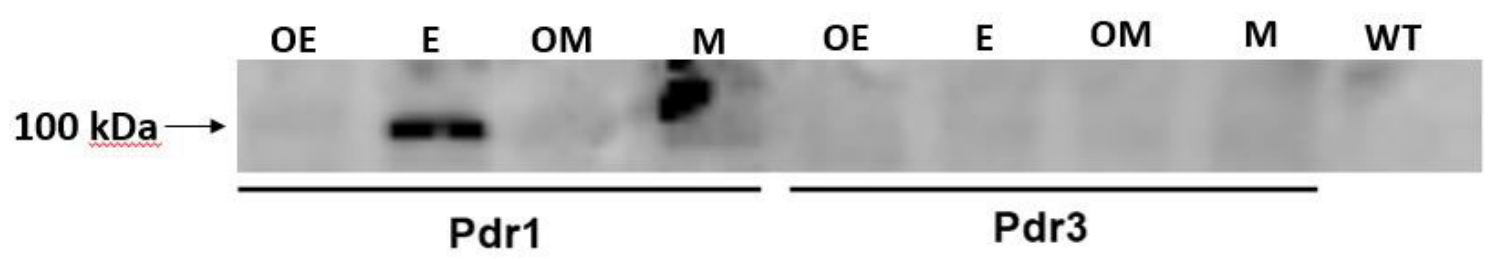

Figure 4. Immunoprecipitation of Pdr1-HA and Pdr3-HA of cells treated with ethanol only (E) or with menadione dissolved in ethanol (M). Only Pdr1 was detectable in a band visible at approximately $100 \mathrm{kDa}$. OE and OM were the control samples from an empty wild-type strain.

Pdr1 in this case was clearly visible, as expected in a band at around $100 \mathrm{kDa}$ mainly in the ethanol sample. Menadione treatment seems to decrease the amount of detectable Pdr1 protein. It may be due to the time interval of the incubation. This hypothesis is investigated further in the following experiments. Pdr3, even after immunoprecipitation, was not detectable, which might reflect its poor abundance in the cell. With the results obtained, the project was continued with a focus on Pdr1 treated with menadione.

\section{Detection of Pdr1 during treatment with menadione and ethanol control using exponential cell growth}

The previous results demonstrated that Pdr1 protein levels were sufficiently high to investigate a possible regulation by xenobiotics using Western blot in whole protein extracts. The goal was to distinguish effects from the organic solvent alone (either ethanol or DMSO) compared to menadione. Cells were treated with different agents and Pdr1 levels were determined in a kinetic manner. Pdr1 levels were clearly visible on the blot before ethanol treatment and continuously decrease over time, whereas at the time point of 4 hours (240 minutes) Pdr1 almost disappears completely as seen in Figure 5. Pdr1 detection after menadione exposure in the same time spans remains strong, and after 4 hours band is still visible on the blot in Figure 6 . The reaction of ethanol treatment and sample behaviour can be compared with the total protein results in Figure 7. Pdr1 bands were visible at expected molecular weight at around $100 \mathrm{kDa}$. 


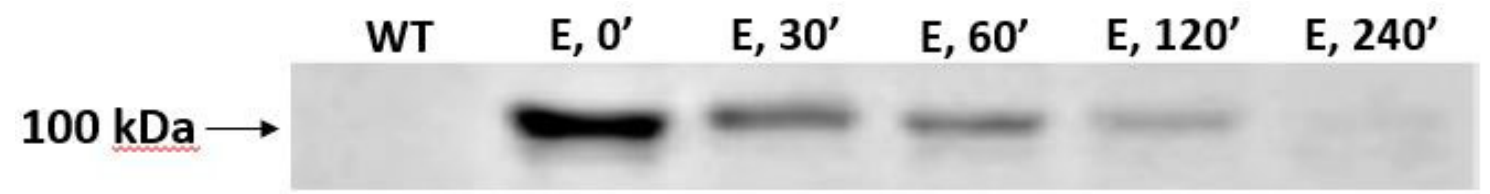

Figure 5. Pdr1-HA levels detected by Western blot, with ethanol treatment alone. Kinetics applied from time point zero to 4 (240') hours of exposing cells to the xenobiotics.

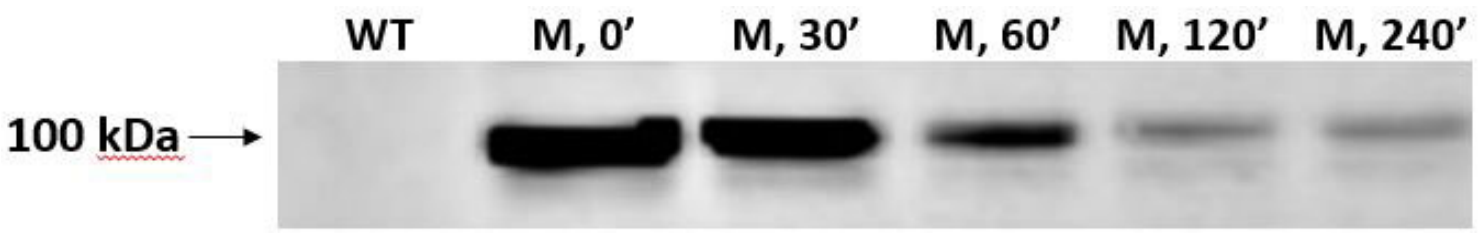

Figure 6. Pdr1-HA levels detected by Western blot with menadione + ethanol treatment. Kinetics applied from time point zero to 4 hours of exposure.

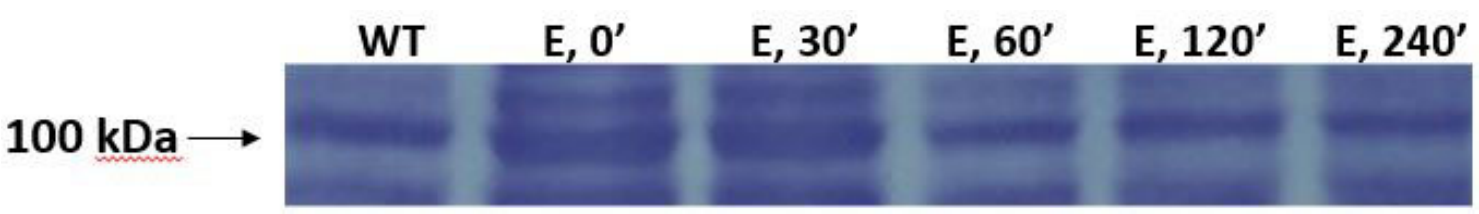

Figure 7. Results of electrophoresis, total cell protein load visualized on PVDF membrane with DB71 staining. Proteins bands were visible in the molecular weight range indicated at around $100 \mathrm{kDa}$. Kinetics applied from time point zero to 4 hours of exposure.

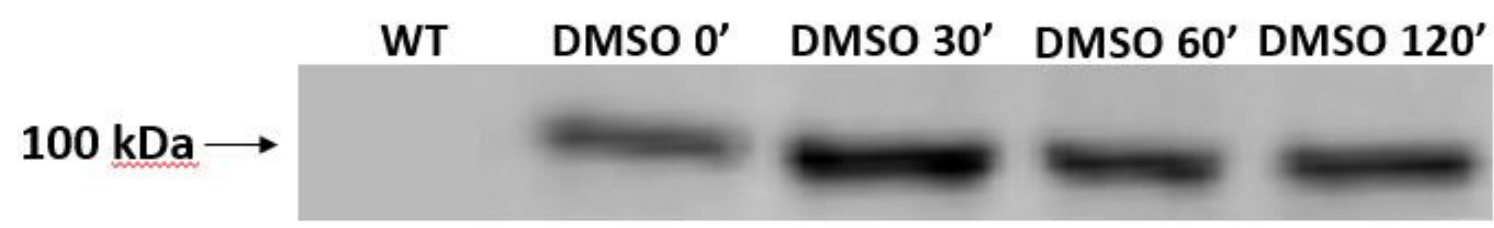

Figure 8. Pdr1-HA levels detected by Western blot treatment with the solvent DMSO alone. Kinetics applied from time point zero to 2 hours of exposure.

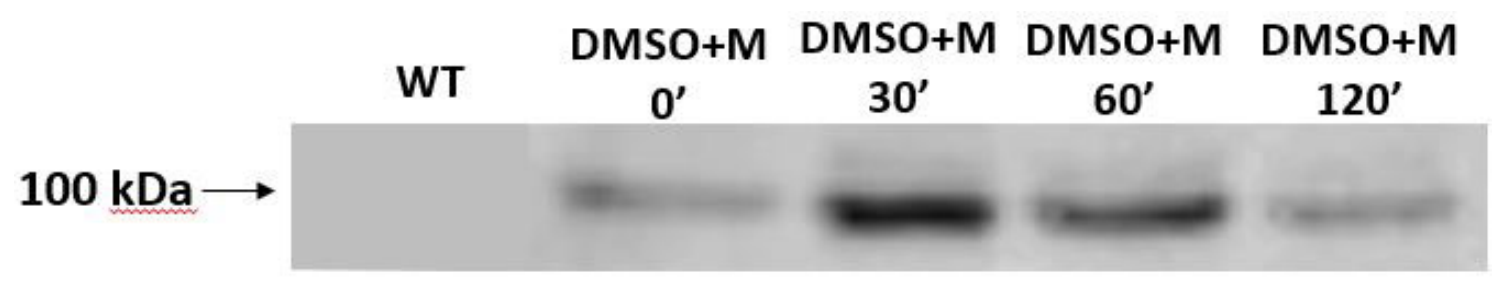

Figure 9. Pdr1-HA levels detected by western blot during the treatment with menadione + DMSO. Kinetics applied from time point zero to 2 hours of exposure.

Detection of Pdr1 during the exposure with menadione and DMSO using exponential cell growth

The previous experiments indicated a decrease of Pdr1 levels in response to both ethanol (solvent) and menadione + ethanol exposure. Therefore, the question was if the application of a different solvent could help to see menadione-specific effects on Pdr1 levels. More specifically, if ethanol, as the solvent for menadione in the previous experiment had any influence in the whole process. In the following experiments, the change of solvent to DMSO was performed and compared its effects. Applying time points from time zero to 
120 minutes. The timepoint at this part of the experiment was shortened since it was seen in previous results that there is no significant difference in duration of the experiment after 120 minutes. The results with DMSO-only were represented in Figure 8. While the results containing menadione dissolved in DMSO can be seen in Figure 9.

In this case, we observed a clear difference between the solvent-only treatment and the menadione treatment. DMSO alone did not seem to alter the intracellular Pdr1 protein levels. The DMSO alone had a continuous reaction of TFs to oxidative stress. The prolonged reaction could be due to organic properties of DMSO. However, after menadione exposure, we detected a brief increase of the protein levels until $30 \mathrm{~min}$ which was followed by a continuous decline of Pdr1 levels. It could be that while Pdr1 is engaged in actively recognizing menadione, it is induced. After xenobiotic recognition, it seems that Pdr1 diminishes. It could mean a proteolytic reduction of the factor after the immediate menadione defence. Therefore, as the Pdr1 effects are apparent, the next interesting question would be if changes in Pdr1 levels could be seen when it is bound to DNA. To find an answer to that question, we performed in vivo chromatin immunoprecipitation experiments with Pdr1-HA and the PDR5 and SNQ2 target genes using quantitative PCR.

\section{Real-time quantitative PCR detecting Pdr1-HA binding to the PDR5 and SNQ2 genes}

Here we performed a kinetic analysis of DNA binding of the Pdr1 TF to two different target genes, SNQ2 and PDR5. PDR5 and SNQ2 are both regulated by Pdr1 and directly targeted by Pdr1 in the promoter region. Menadione and/or ethanol treatments were applied to follow the amount of DNA-bound Pdr1 using chromatin immunoprecipitation. Results are visible in Figure 10 and Figure 11 where Pdr1 seems to be more present at the PDR5 and SNQ2 target promoters when treated with menadione comparing to ethanol samples, especially at 40 minutes.

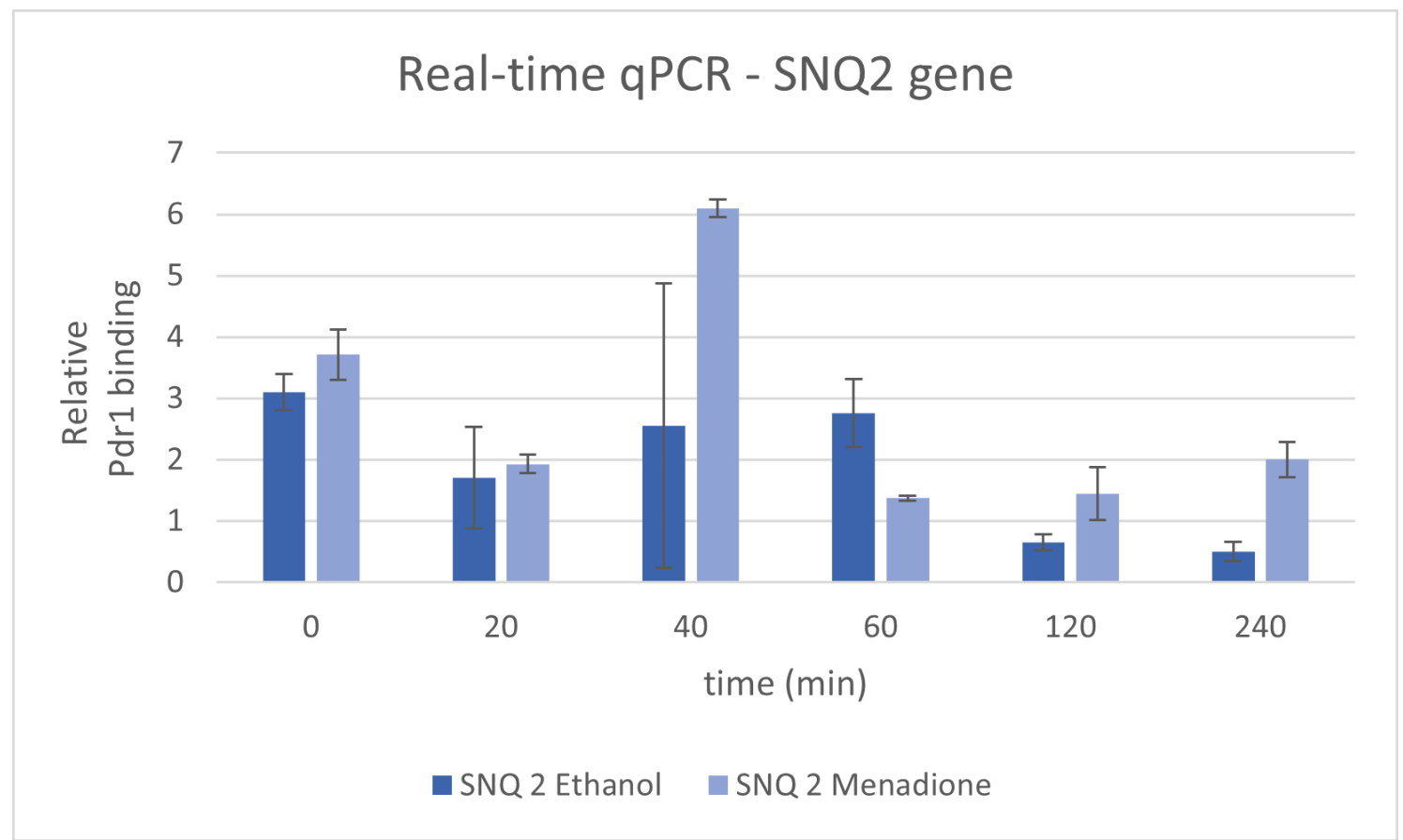

Figure 10. ChIP-PCR analysis of Pdr1-HA binding to SNQ2 gene. Results after menadione and ethanol treatment (Performed in triplicates). 


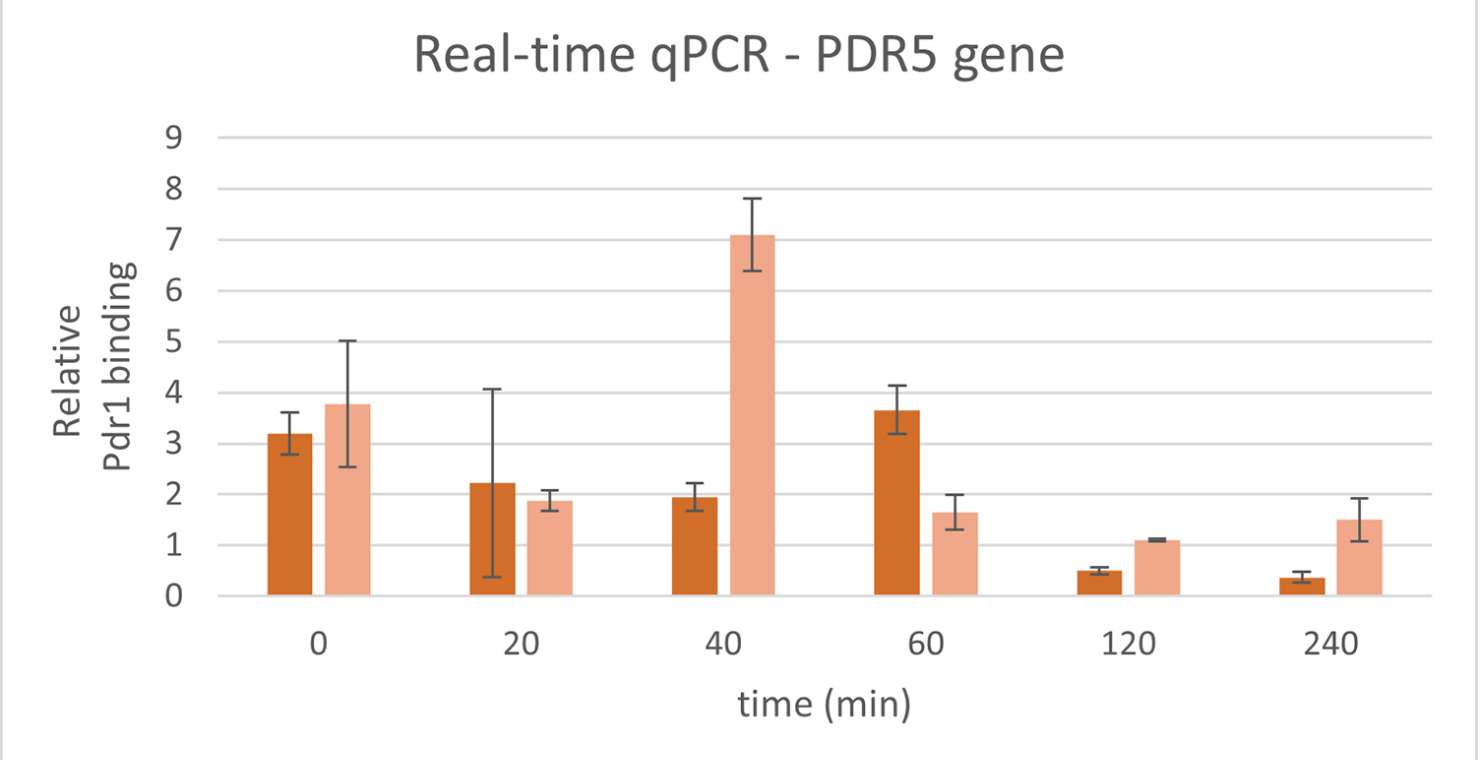

- PDR 5 Ethanol aPD 5 Menadione

Figure 11. ChIP-PCR analysis of Pdr1-HA binding to PDR5 gene. Results after menadione and ethanol treatment (Performed in triplicates).

That could mean that Pdr1 at those time points was acting as a transcriptional activator. Nevertheless, after this time points the Pdr1 bound level drastically decreases. In the ethanol control, this up-regulation is not observed. Instead, the level seems to be maintained during time with a slight decrease after 2 hours. This was an obvious difference comparing ethanol (control) and menadione results for the same genes. Looking at the later time points in the control, there was noticeable less binding. It is hard to interpret this with certainty since the experiment is performed for the first time. Further research and experiments should be done. Taken together it seems that after menadione exposure, Pdr1 was increasingly bound at target promoters as an activator until approximately 40 minutes, with a peak at 40 minutes. After $40 \mathrm{~min}$, Pdr1 seems to be removed from chromatin by unknown action. Also, the binding levels at 20 minutes were lower than expected, thus more repetitions or different approach could clarify the kinetics of Pdr1 binding in the early adaptation phase.

\section{Effect of TF overexpression and adaptation to different menadione doses}

All the graphs presented in this experiment can be explained together. Figure 12, Figure 13, and Figure 14 represent certain strains under the influence of menadione treatment, grouped according to cell type over-expression strains treated with all menadione concentrations. The total time of measurement is 93 hours and 30 minutes. The hybrid between the proteins in combination with the Pdr1 and Gal4 was used from the lab collection. The hybrid was formed in a way that the DNA binding domain (DBD) of a prospective protein (in this case Pdr1) was replaced with the DBD of Gal4 TF. Accordingly, we tested the effect of overexpressing the Pdr proteins without their native DNA binding domain and thus not allowing them to activate their natural target genes. To obtain these results, different concentrations of menadione were applied to cell lines over-expressing Pdr1-Gal4, Pdr3- 
Gal4 or Stb5-Gal4. Stb5 was included as an addition to test the hypothesis in contrast to expected reactions of Pdr1 and Pdr3. In a 96 well plate, each overexpressing group was performed in duplicates while introduced to menadione concentrations of $0 \mu \mathrm{M}$ (control), 25 $\mu \mathrm{M}, 50 \mu \mathrm{M}$, and $75 \mu \mathrm{M}$. The mean value is calculated afterwards and presented on graphs. Looking at results, Stb5 overexpression seems to be beneficial (Figure 14). On the other hand, the expected result was more of a gradual decrease with the increase of menadione concentrations evoking stress.

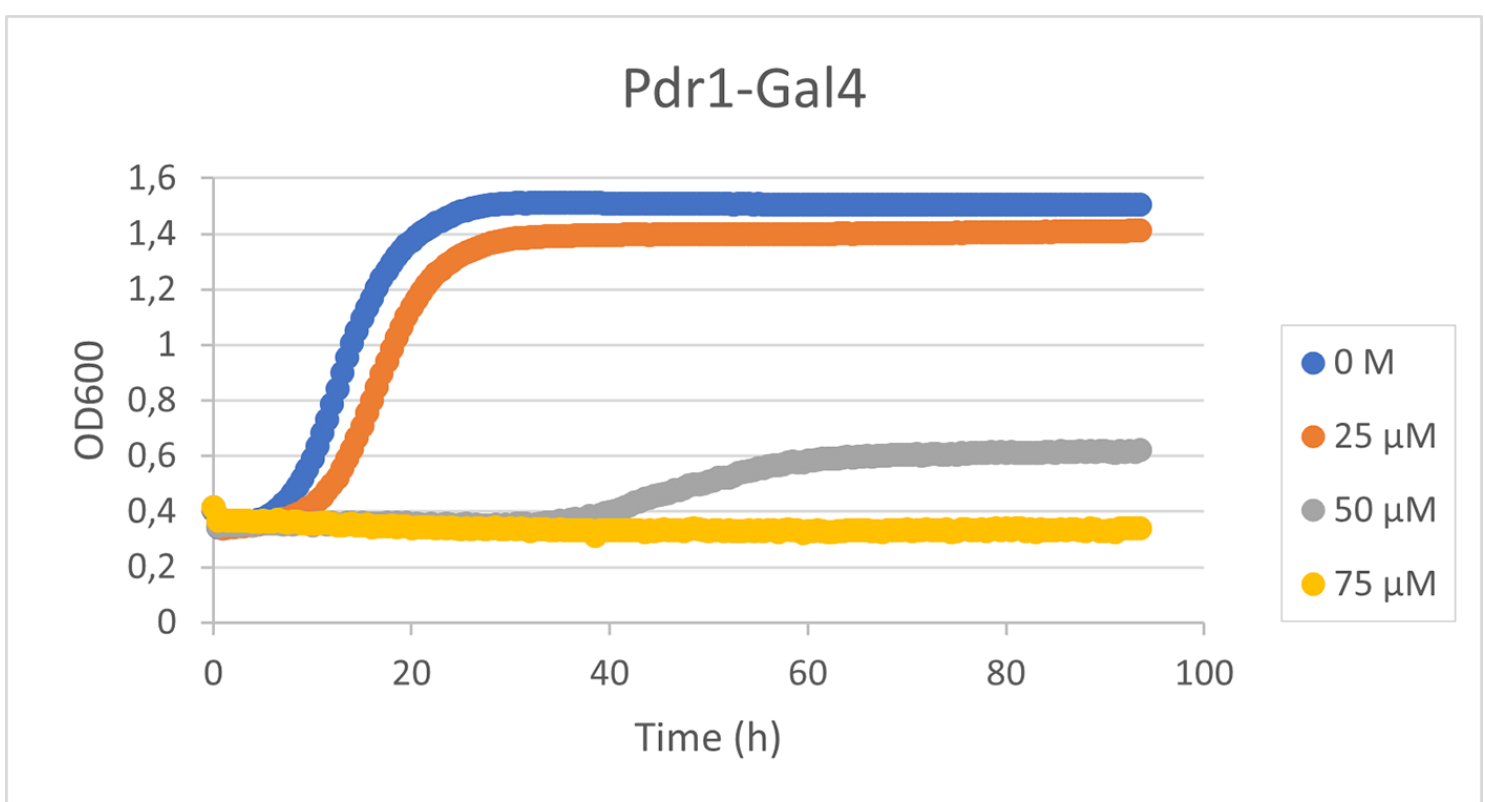

Figure 12. Growth efficiency of Pdr1-Gal4 overexpressing yeast cells in the presence of various menadione concentrations. Representing all Pdr1-Gal4 over-expressions combined.

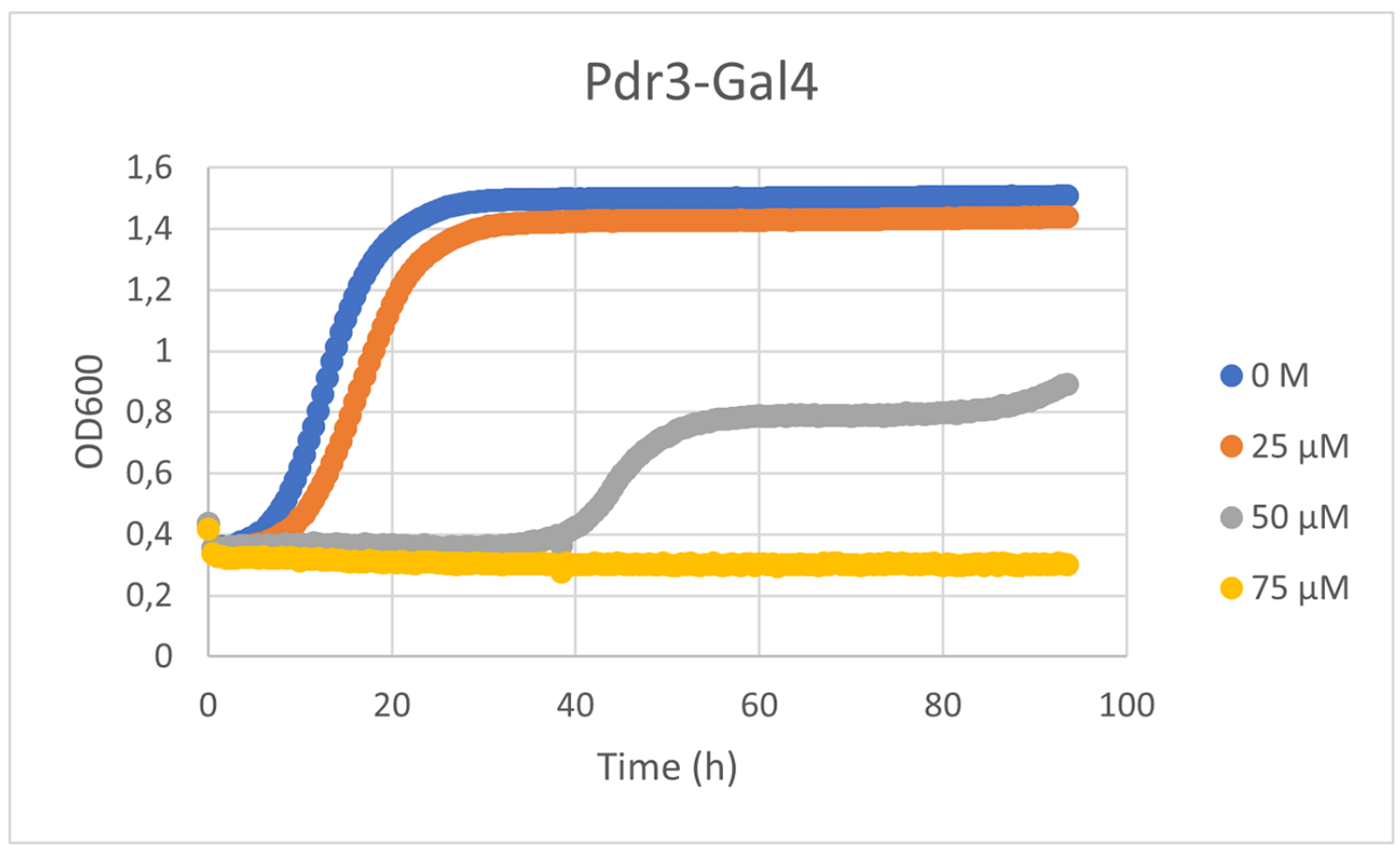

Figure 13. Growth efficiency of Pdr3-Gal4 overexpressing yeast cells in the presence of various menadione concentrations. Representing all Pdr3-Gal4 over-expressions combined. 


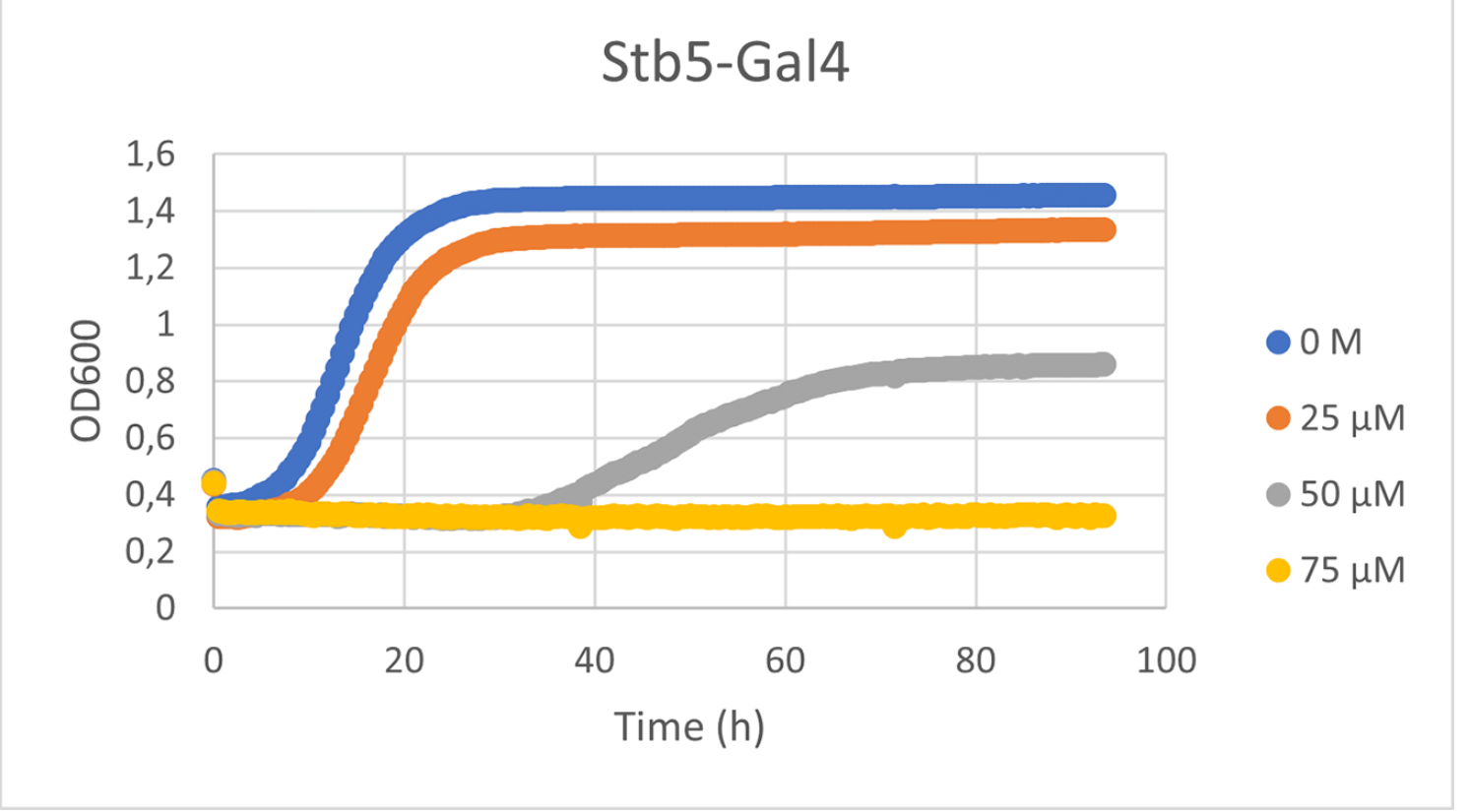

Figure 14. Growth efficiency of Stb5-Gal4 overexpressing yeast cells in the presence of various menadione concentrations. Representing all Stb5-Gal4 over-expressions combined.

\section{Discussion}

In the experiments performed, Pdr1 and Pdr3 transcription activators, belonging to the PDR system of $S$. cerevisiae were targeted and assayed individually regarding their response to xenobiotics (menadione). These early results indicated better expression of the Pdr1 transcriptional factor, compared to its homologous Pdr3. The defence system was also tested against ethanol and DMSO to exclude the possibility of overlaying the effects on the cell. Organic solvents are additional stressors to $S$. cerevisiae. The results indicate that Pdr1 is most abundant and active after 30 minutes from the beginning of the treatment, compared with 240 minutes after the treatment when the function of the transcription factor is faded. Further results indicate that Pdr1 binding to the PDR5 and SNQ2 genes which are both activated by the Pdr1 regulator, peaks at around the same time, or more precisely after 40 minutes from the start of the treatment. The critical point of the PDR system is that transporters are normally not expressed or are at very low levels. When a cell faces chemical stress, it responds by activating one, several, or all the transporters.

Although our results seemed to establish a redundant role of the homologous TFs in the PDR system, more recent research indicates that specificities of Pdr1 and Pdr3 transcriptional factors are different and can vary. One important difference is that Pdr1 is a much more abundant TF when compared to Pdr3. This was also proven in this research. Unlike Pdr3, Pdr1 triggers DNA activation as a reaction to menadione and most probably at first hand detects it. Menadione is not able to efficiently trigger Pdr3 activation (VanacloigPedros, Lozano-Pérez, Alarcón, Pascual-Ahuir, \& Proft, 2019). Importantly, the transcription induced by toxic compounds is the most significant part of assessing the xenobiotic antagonism since antagonistic strains of morbific $S$. cerevisiae are related to pathogenic yeast in general (Moye-Rowley, 2019; Cavalheiro et al., 2019; Healey \& Perlin, 2018; Vanacloig-Pedros et al., 2019). According to the literature, specific linkage experiments were implemented in vitro containing Pdr1 and its homologous Pdr3 TF. The results have 
indicated that both transcriptional factors have the potential to link various structural xenobiotics, including rifampicin, cycloheximide, or antifungal azoles (Vanacloig-Pedros et al., 2019; Thakur et al., 2008).

Pdr1 seems to undergo fluctuations in abundance when treated with a target compound. A possible explanation is that menadione binding to Pdr1 first converts Pdr1 in an activator (also seen in qPCR results), but then Pdr1 returns to background activity. This might be achieved by its degradation, because hydrophobic binding of menadione to Pdr1 might not be easily reversible. However, this concept alone is not sufficient for the detoxification of Pdr1 XBD alone, as seen in the growth experiment. The detection and recognition of the compound happens at the discrete xenobiotic binding domain at the middle portion of the protein (Figure 15).

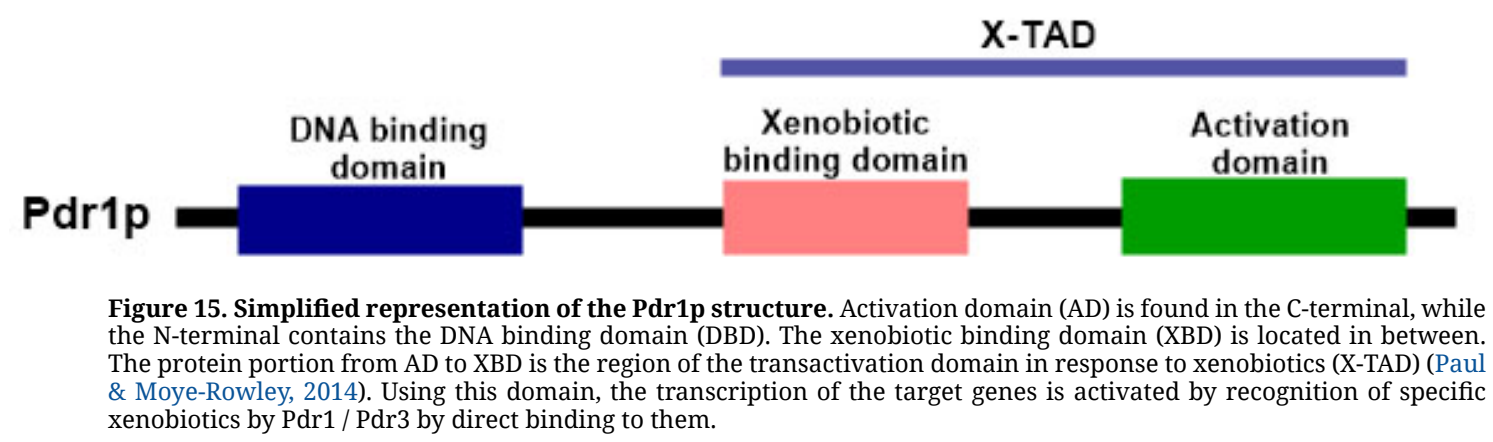

Given the fact that Pdr1 directly binds the xenobiotic molecule, its destruction might help the cells to remove toxic levels of menadione. It could be possible that overexpressing the part of Pdr1 which recognizes menadione alone was sufficient to detoxify and hence produce a tolerance towards menadione. To this end, the advantage was taken of recently developed yeast strains overexpressing Pdr1-Gal4 fusion proteins. In this case, the native DNA-binding domain (DBD) of Pdr1 has been replaced by the DNA binding domain (DBD) of the Gal4 transcription factor. Figure 15 shows the general structure of the Pdr1 protein with XBD.

Pdr1 and Stb5 were able to recognize and directly bind menadione. The hybrid Pdr1-Gal4 protein still recognizes menadione and gets activated as a transcription factor, however, it does no longer promote the induction of the natural target genes, namely numerous xenobiotic-transporter encoding genes. Additionally, we included alternative overexpressing strains: Pdr3-Gal4, which does not identify menadione, and Stb5-Gal4, which moderately responds to menadione (Vanacloig-Pedros et al., 2019). If results were as expected; the Pdr1-Gal4 overexpression strain would resist menadione better or grow better with higher menadione concentration when compared to other strains, but that didn't seem to happen. Obtained results indicate that Pdr1-Gal4 overexpression seems to slightly delay the growth with menadione. Stb5 has grown slightly better when compared to Pdr1, with Pdr3 unexpectedly showing the same growth efficiency. By conducting this experiment, it is possible to exclude the fact that overexpression of the Pdr1 xenobiotic recognition domain alone is not helping the cells to tolerate high menadione concentrations. 
The experiment is correctly executed, as the inhibition of the strains by menadione is visible. However, in this certain condition, it is not visible that Pdr1-Gal4 overexpression rescues cell growth. According to the results, this complex topic could be further studied and is probably not as simple as proposed - that when we overexpress the Pdr1 XBD using Pdr1-Gal4 strain, the toxic effect of menadione is removed. This was an alternative strategy to the conventional approach of overexpressing native Pdr1 or several xenobiotic transporters, which clearly improves the growth of the cells in the presence of harmful xenobiotic concentrations. Taken together, the overexpression of xenobiotic binding TFs without their native DNA binding capability is not sufficient to create resistance.

Pdr1 and Pdr3 both have an elevated level of sequence conservation among all transcriptional factors of the "Pdr like" group in S. cerevisiae. Nevertheless, there is only $37 \%$ similarity in their XBDs. That leads to the hypothesis that the different Pdr TFs have developed certain divergent identification patterns throughout evolution (Vanacloig-Pedros et al., 2019). A non-redundant function for the TFs of interest, in this case, Pdr3 and Pdr1 have been proposed before for xenobiotics that do not need Pdr1 but require Pdr3 for defence when it comes to toxic aliphatic solvents (Nishida-Aoki, Mori, Kuroda, \& Ueda, 2015). Regarding the Pdr1 and Pdr3 homology and the distinctive reaction to menadione, the further hypothesis could be set on determining detailed differences in XBDs of both TFs regarding menadione. Another interesting question appearing here is, how do they mutually interact when binding to DNA and does the one with the higher affinity takes over the transcription. Further experiments could explain the position of the TFs and correlation to XBD and its PDRE motifs. In addition, a strategy was found that can serve to increase the efficiency of treatment in fungal infection (Thakur et al., 2008).

On the other hand, it is interesting to point out that the genomic resemblance does not play a significant role when it comes to an analogous compound identification of transcription factors of interest, since the study of Vanacloig-Pedros et al. (2019) provided results of observing structured transactivation of Yrr1 and Pdr1 via the toxic ochratoxin A molecule. Should be kept in mind that both of those TFs share a significantly decreased amount of resemblance than Pdr3 TF and Pdr1 TF do. Previous studies conducted on the topic pointed out massive use of the effects obtained in mutant strains lacking specific components belonging to MDR, such as tolerance or resistance to certain xenobiotic treatments. However, Pdr TFs have to be investigated directly to prove their function within the multidrug system under normal genetic conditions. Nevertheless, those essentially operating Pdr TFs, mainly Pdr1, Pdr3 and Yrr1; indicate a drug-specific gene activation of the plasma membrane transporters (Zhang, Cui, Miyakawa, \& Moye-Rowley, 2001; Keeven, Ko, Shallom, Uccelini, \& Golin, 2002; Rong-Mullins, Ayers, Summers, \& Gallagher, 2018). Methods applied in this project can quantify the identification of the compound by single transcription factors and can contribute to continuing research into various unicellular or multicellular organism's drug recognizing transcription factors.

To summarize, the defence system of yeast is a group of well-defined and distinctive transcriptional activators. For that reason, to investigate the hypothesis, further research could be done. It would be interesting to observe the behaviour of the overexpressing Pdr1 XBD when reacting with other toxins, besides menadione. On the other hand, time 
points seemed to play a crucial role in detecting the transcription in action. Possibly, more detailed observation could bring some new insights into the efficient activation of gene transcription.

Provenance: Submitted. Based on a Master Thesis of Tea Vasiljević, Submitted to Bonn-Rhein-Sieg University of Applied Sciences, Department of Natural Sciences (Rheinbach, Germany) for the degree of Master of Science in Biomedical Sciences, 2019. Thesis was made at the Institute of Biomedicine of Valencia, CSIC (Valencia, Spain), Department of Molecular and Cellular Pathology and Therapy, Laboratory for Cellular Homeostasis and Adaptation to Stres.

Peer review: Externally peer reviewed.

Received: 17 April 2021 / Accepted: 2 September 2021 / Published online: 24 September 2021.

Funding: This research received no specific grant from any funding agency in public, commercial or not-for-profit sectors.

Acknowledgements: Many thanks to PhD student Josep Fita Torro for all the patience and help with mastering my lab techniques. Appreciate that Instituto de Biomedicina de Valencia gave me the placement to carry out my work.

Authorship declaration: TV performed and conducted the experiments using molecular techniques, statistical analysis, and interpretation of the results, drafting the manuscript and literature review. MP designed the study, supervised the process of experiment and results in conduction, editing of the manuscript. TV and MP read and approved the final version of the manuscript.

Competing interests: The authors completed the ICMJE Unified Competing Interest form (available upon request from the corresponding author), and declare no conflicts of interest.

\section{ORCID}

Tea Vasiljević (iD https://orcid.org/0000-0001-7195-6219

Markus Proft (10) https://orcid.org/0000-0002-6788-5830

\section{References}

Akache, B., Wu, K., \& Turcotte, B. (2001). Phenotypic analysis of genes encoding yeast zinc cluster proteins. Nucleic Acids Research, 29:2181-2190.

Balzi, E., Chen, W., Ulaszewski, S., Capieaux, E., \& Goffeau, A. (1987). The multidrug resistance gene PDR1 from Saccharomyces cerevisiae. The Journal of biological chemistry, 262(35), 16871-16879.

Balzi, E., \& Goffeau, A. (1995). Yeast multidrug resistance: the PDR network. Journal of Bioenergetics and Biomembranes, 27:71-76.

Cavalheiro, M., Costa, C., Silva-Dias, A., Miranda, I. M., Wang, C., Pais, P., Pinto, S. N., Mil-Homens, D., Sato-Okamoto, M., Takahashi-Nakaguchi, A., Silva, R. M., Mira, N. P., Fialho, A. M., Chibana, H., Rodrigues, A. G., Butler, G., \& Teixeira, M. C. (2018). A Transcriptomics Approach To Unveiling the Mechanisms of In Vitro Evolution towards Fluconazole Resistance of a Candida glabrata Clinical Isolate. Antimicrobial agents and chemotherapy, 63(1), e00995-18.

Chen, K., Miyazaki, T., Tsai, H. \& Bennett, J. (2007). The bZip transcription factor Cgap1p is involved in multidrug resistance and required for activation of multidrug transporter gene CgFLR1 in Candida glabrata. Gene, 386(1-2), pp.63-72.

Delaveau, T., Delahodde, A., Carvajal, E., Subik, J., \& Jacq, C. (1994). PDR3, a new yeast regulatory gene, is homologous to PDR1 and controls the multidrug resistance phenomenon. Molecular Genetics and Genomics, 244, 501-511. 
Healey, K. R., \& Perlin, D. S. (2018). Fungal Resistance to Echinocandins and the MDR Phenomenon in Candida glabrata. Journal of fungi (Basel, Switzerland), 4(3), 105.

Hong, H. Y., Yoo, G. S., \& Choi, J. K. (2000). Direct Blue 71 staining of proteins bound to blotting membranes. Electrophoresis, 21, 841-845.

Jungwirth, H., \& Kuchler, K. (2006). Yeast ABC transporters - A tale of sex, stress, drugs and aging. FEBS letters, 580(4), 1131-1138.

Katzmann, D. J., Burnett, P. E., Golin, J., Mahe, Y., \& Moye-Rowley, W. S. (1994). Transcriptional control of the yeast PDR5 gene by the PDR3 gene product. Molecular and cellular biology, 14(7), 46534661.

Keeven, J., Ko, D., Shallom, J., Uccelini, B., \& Golin, J. (2002). PDR2 Gain-of-function mutations eliminate the need for Pdr1 and require the UBP6 product for resistance to translational inhibitors. Current Genetics, 41, 11-19.

Kuras, L., \& Struhl, K. (1999). Binding of TBP to promoters in vivo is stimulated by activators and requires Pol II holoenzyme. Nature, 399(6736):609-13.

Leppert, G., McDevitt, R., Falco, S. C., Van Dyk, T. K., Ficke, M. B., \& Golin, J. (1990). Cloning by gene amplification of two loci conferring multiple drug resistance in Saccharomyces. Genetics, 125:13-20.

Löscher, W., \& Potschka, H. (2005). Drug resistance in brain diseases and the role of drug efflux transporters. Nature reviews. Neuroscience, 6(8):591-602.

Mamnun, Y. M., Pandjaitan, R., Mahé, Y., Delahodde, A., \& Kuchler, K. (December 2002). “The yeast zinc finger regulators Pdr1p and Pdr3p control pleiotropic drug resistance (PDR) as homo- and heterodimers in vivo”. Molecular Microbiology, 46 (5): 142940.

Meyers, S., Schauer, W., Balzi, E., Wagner, M., Goffeau, A., \& Golin, J. (1992). Interaction of the yeast pleiotropic drug resistance genes PDR1 and PDR5. Current Genetics, 21:431.

Moye-Rowley, W. S. (2019). Multiple interfaces control activity of the Candida glabrata Pdr1 transcription factor mediating azole drug resistance. Current Genetics, 65, 103 -108.

Nishida-Aoki, N., Mori, H., Kuroda, K., \& Ueda, M. (2015). Activation of the mitochondrial signaling pathway in response to organic solvent stress in yeast. Current Genetics, 61, 153-164.

Paul, S., \& Moye-Rowley, W. S. (2014). Multidrug resistance in fungi: regulation of transporterencoding gene expression. Frontiers in physiology, 5:143.

Prasad, R., \& Rawal, M. K. (2014). Efflux pump proteins in antifungal resistance. Frontiers in pharmacology, 5:202.

Rank, G. H., \& Bech-Hansen, N. T. (1973). Single nuclear gene inherited cross resistance and collateral sensitivity to 17 inhibitors of mitochondrial function in S. cerevisiae. Molecular \& general genetics, 126:93-102.

Rong-Mullins, X., Ayers, M. C., Summers, M., \& Gallagher, J. E. G. (2018). Transcriptional Profiling of Saccharomyces cerevisiae Reveals the Impact of Variation of a Single Transcription Factor on Differential Gene Expression in 4NQO, Fermentable, and Nonfermentable Carbon Sources. G3: Genes | Genomes | Genetics, 8(2), 607-619.

Sá-Correia, I., dos Santos, S. C., Teixeira, M. C., Cabrito, T. R., \& Mira, N. P. (2009). Drug: H+ antiporters in chemical stress response in yeast. Trends in microbiology, 17(1), 22-31.

Schnell, N., Krems, B., \& Entian, K. (1992). The PAR1 (YAP1/SNQ3) gene of Saccharomyces cerevisiae, ac-jun homologue, is involved in oxygen metabolism. Current Genetics, 21(4-5), pp.269-273.

Thakur, J. K., Arthanari, H., Yang, F., Pan, S. J., Fan, X., Breger, J., Frueh, D. P., Gulshan, K., Li, D. K., Mylonakis, E., Struhl, K., Moye-Rowley, W. S., Cormack, B. P., Wagner, G., \& Näär, A. M. (2008). A nuclear receptor-like pathway regulating multidrug resistance in fungi. Nature, 452(7187), 604-609.

Vanacloig-Pedros, E., Lozano-Pérez, C., Alarcón, B., Pascual-Ahuir, A., \& Proft, M. (2019). Live-cell assays reveal selectivity and sensitivity of the multidrug response in budding yeast. The Journal of biological chemistry, 294(35), 12933-12946.

Zhang, X., Cui, Z., Miyakawa, T., \& Moye-Rowley, W. S. (2001). Cross-talk between transcriptional regulators of multidrug resistance in Saccharomyces cerevisiae. The Journal of biological chemistry, 276, 8812- 8819. 